Supporting Information for

\title{
Amino acid-based Cholinium Ionic Liquids as sustainable catalysts for PET depolymerization
}

Salvatore Marullo, ${ }^{a}$ Carla Rizzo, ${ }^{a}$ Nadka T. Dintcheva, ${ }^{b}$ Francesca D’Anna ${ }^{* a}$

[a] Dipartimento di Scienze Biologiche, Chimiche e Farmaceutiche, Sezione di Chimica, Università degli Studi di Palermo, Viale delle Scienze Ed. 17, 90128 Palermo (Italy)

[b] Dipartimento di Ingegneria, Università degli Studi di Palermo, Viale delle Scienze Ed. 6, 90128 Palermo (Italy)

Corresponding author: Prof. Francesca D’Anna, francesca.danna@unipa.it

Pages: 9

Figures: 3

Tables: 4 


\section{Table of contents}

Figure S1. Representative pictures of post-consumer PET waste used as starting materials.

Figure S2. Expansion of ${ }^{1} \mathrm{H}$ NMR spectra of a solution $[\mathrm{Ch}][\mathrm{Gly}]$ and EG, before and after being heated at $150^{\circ} \mathrm{C}$ for $6 \mathrm{~h}$ or at $180^{\circ}$ for $6 \mathrm{~h}$.

Figure S3. ${ }^{1} \mathrm{H}$ NMR spectra of BHET obtained from glycolysis of post-consumer PET waste.

Table S1. Conversions, yields and reaction conditions for the glycolysis of PET at different temperatures.

Table S2. Conversions, yields and reaction conditions for the glycolysis of PET at different reaction times.

Table S3. Conversions, yields and reaction conditions for the glycolysis of PET at different catalyst loading.

Table S4. Conversions, yields and reaction conditions for the glycolysis of PET promoted by different cholinium salts. 

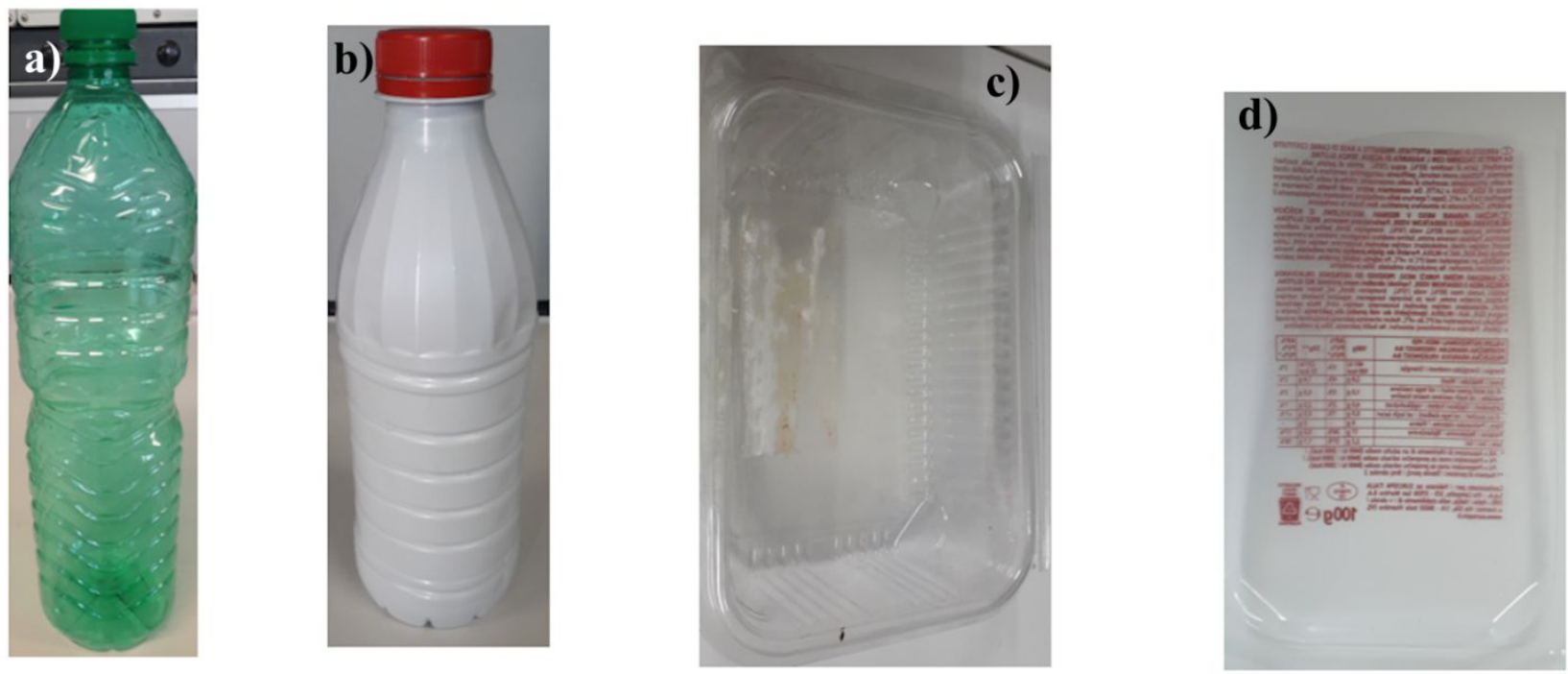

Figure S1. Pictures of plastic waste bottles and containers used for PET depolymerization: a) colored transparent bottle, b) opaque bottle, c) bakery package and d) processed meat package.

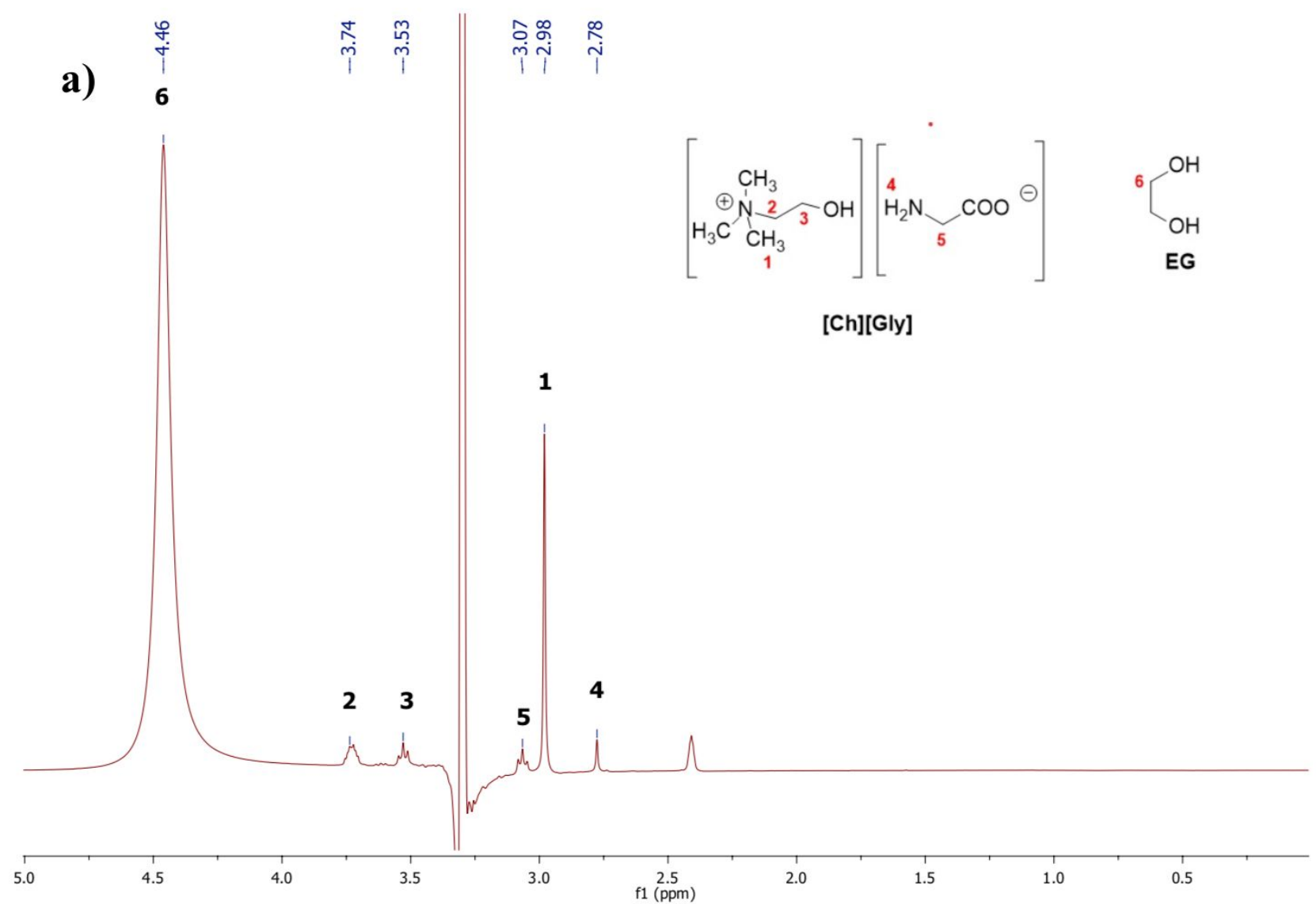



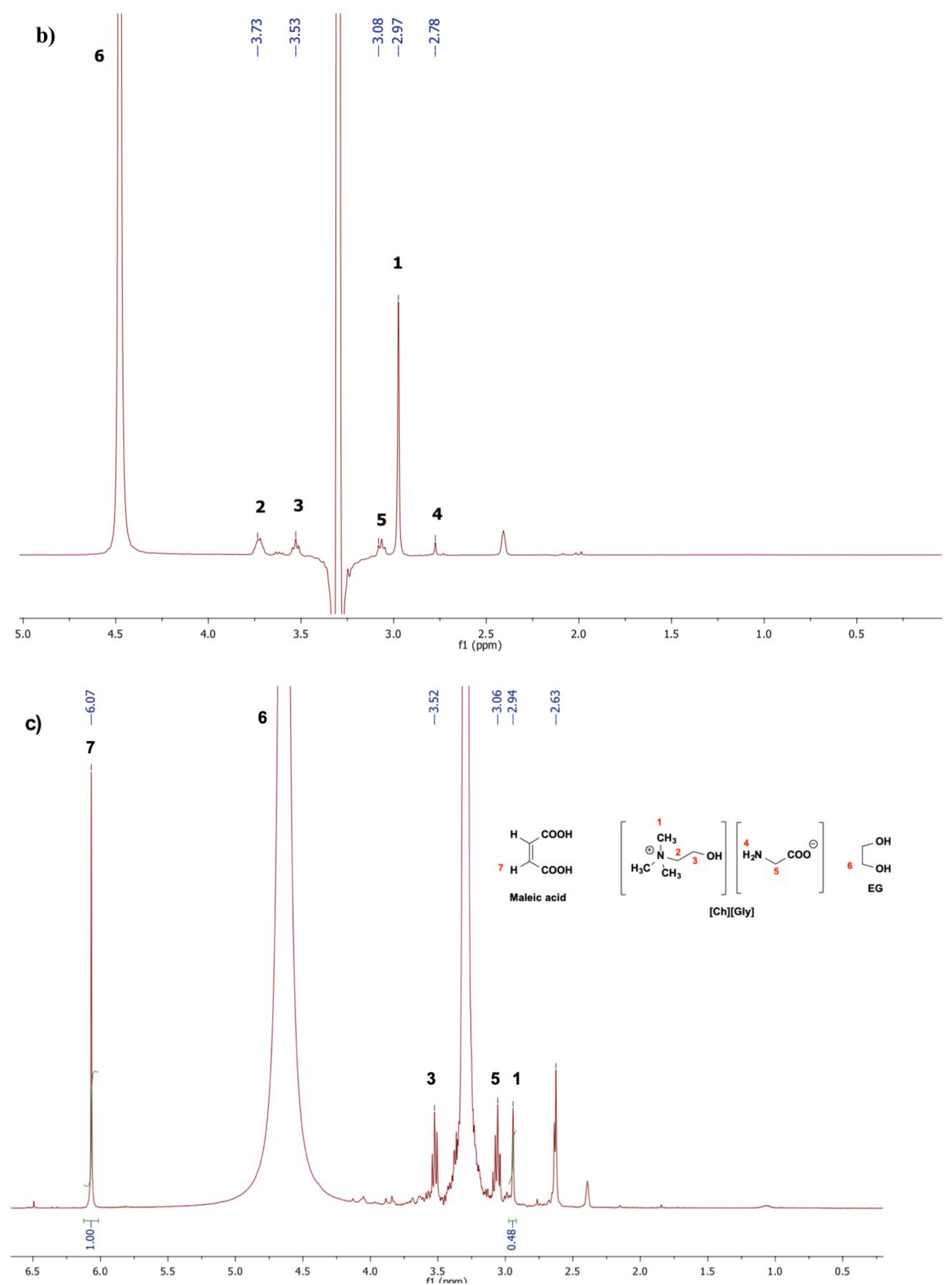

Figure S2. a) Expansion of ${ }^{1} \mathrm{H}$ NMR spectra of $[\mathrm{Ch}][\mathrm{Gly}]$ in $\mathrm{EG}$; b) expansion of the ${ }^{1} \mathrm{H}$ NMR spectrum of the previous solution, after heating at $150{ }^{\circ} \mathrm{C}$ for $6 \mathrm{~h}$; c) expansion of the ${ }^{1} \mathrm{H}$ NMR spectrum of the previous solution, after heating at $180^{\circ} \mathrm{C}$ for $6 \mathrm{~h}$ with added maleic acid as internal standard. Spectra recorded in $\mathrm{d}_{6}$-DMSO. 


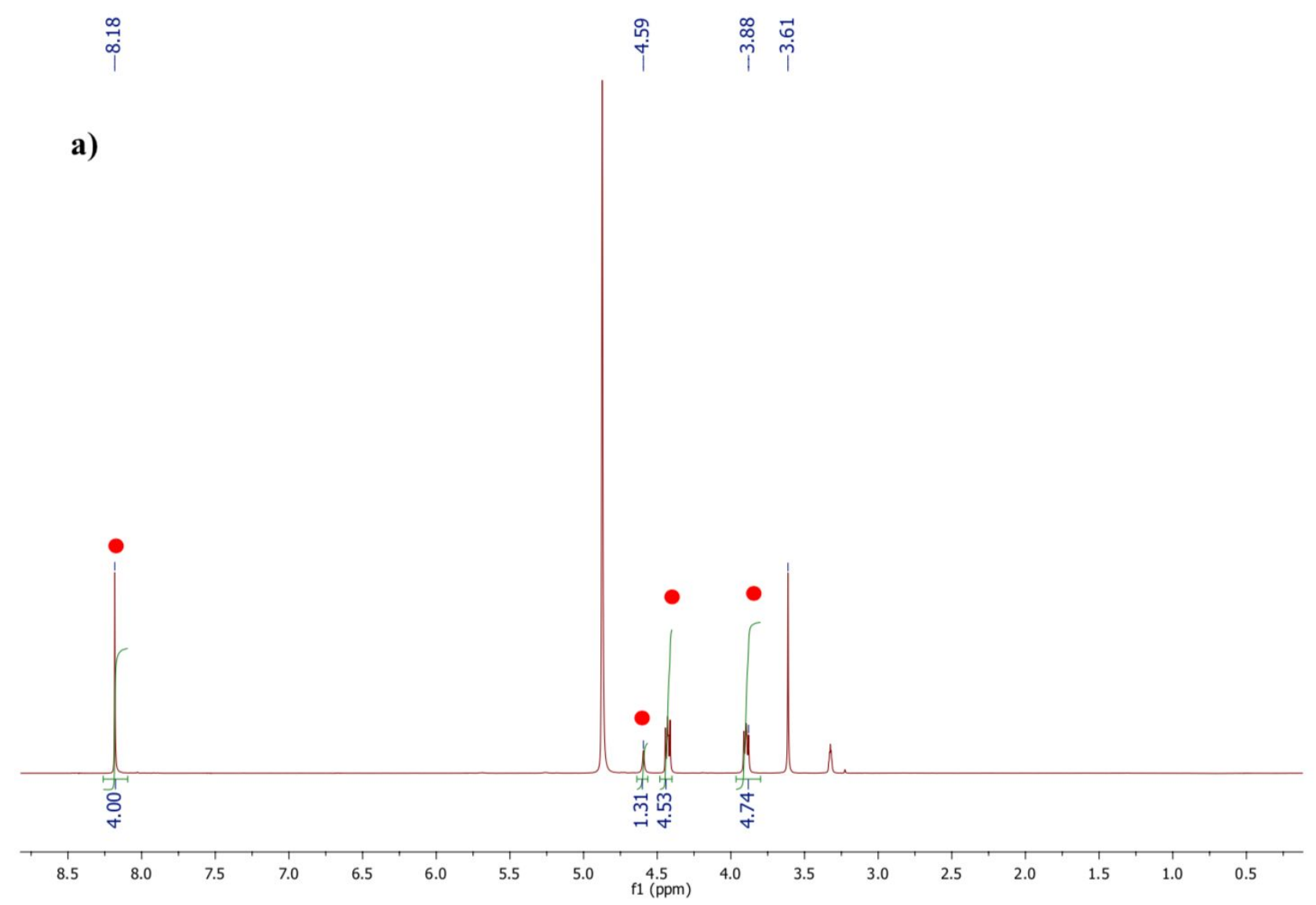

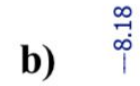
चே

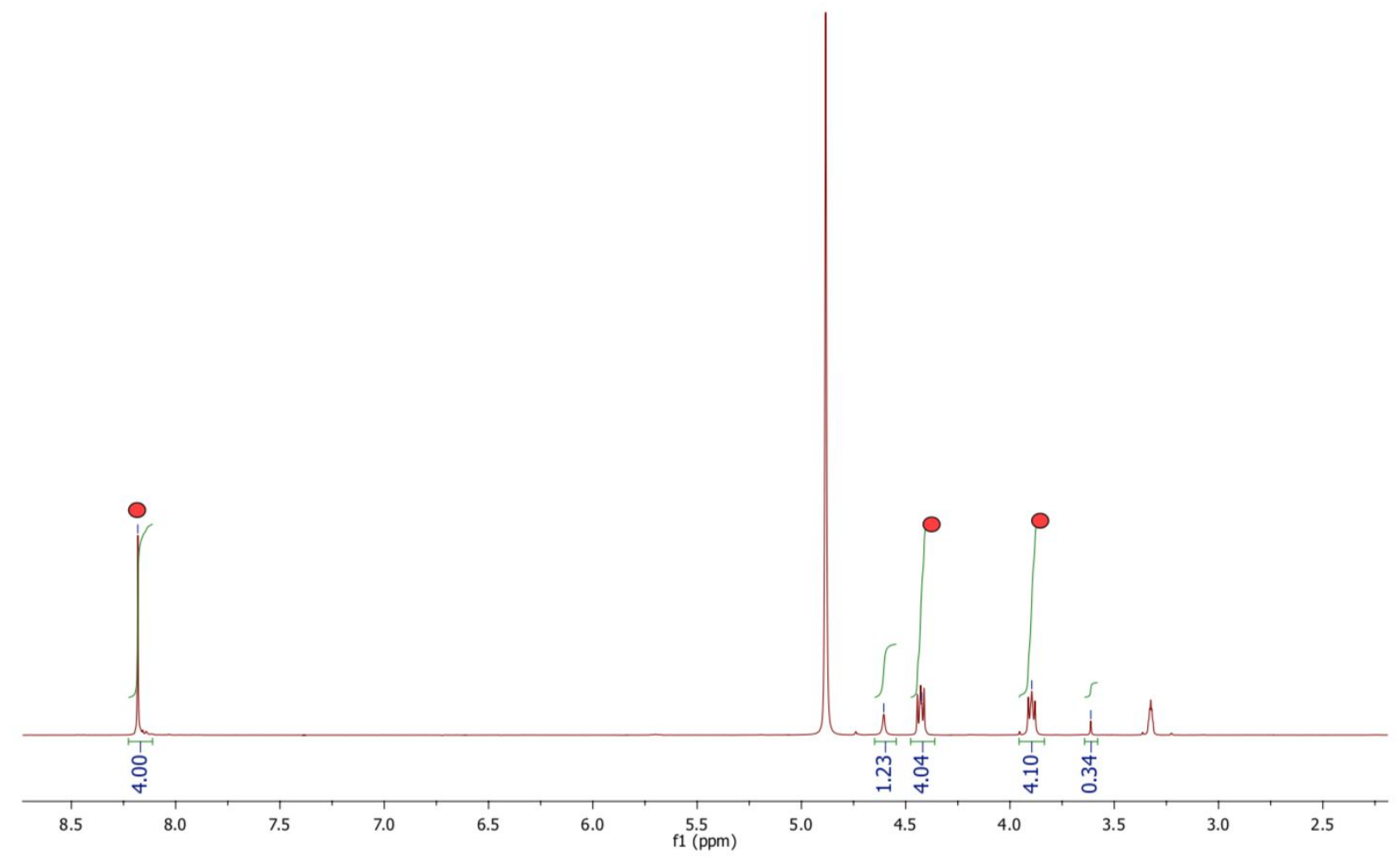



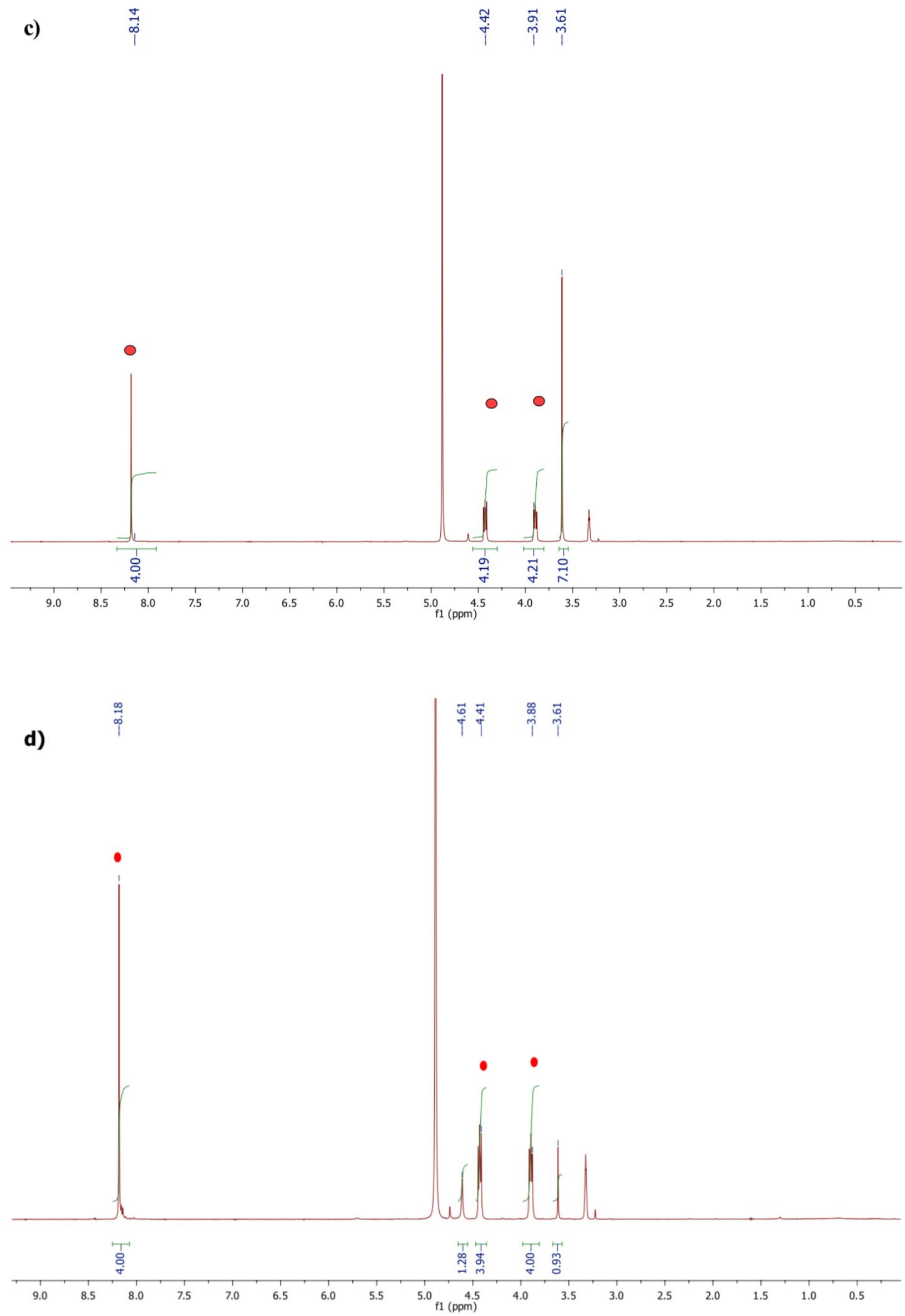


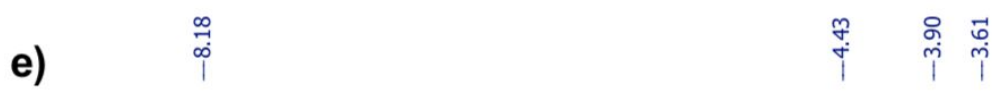

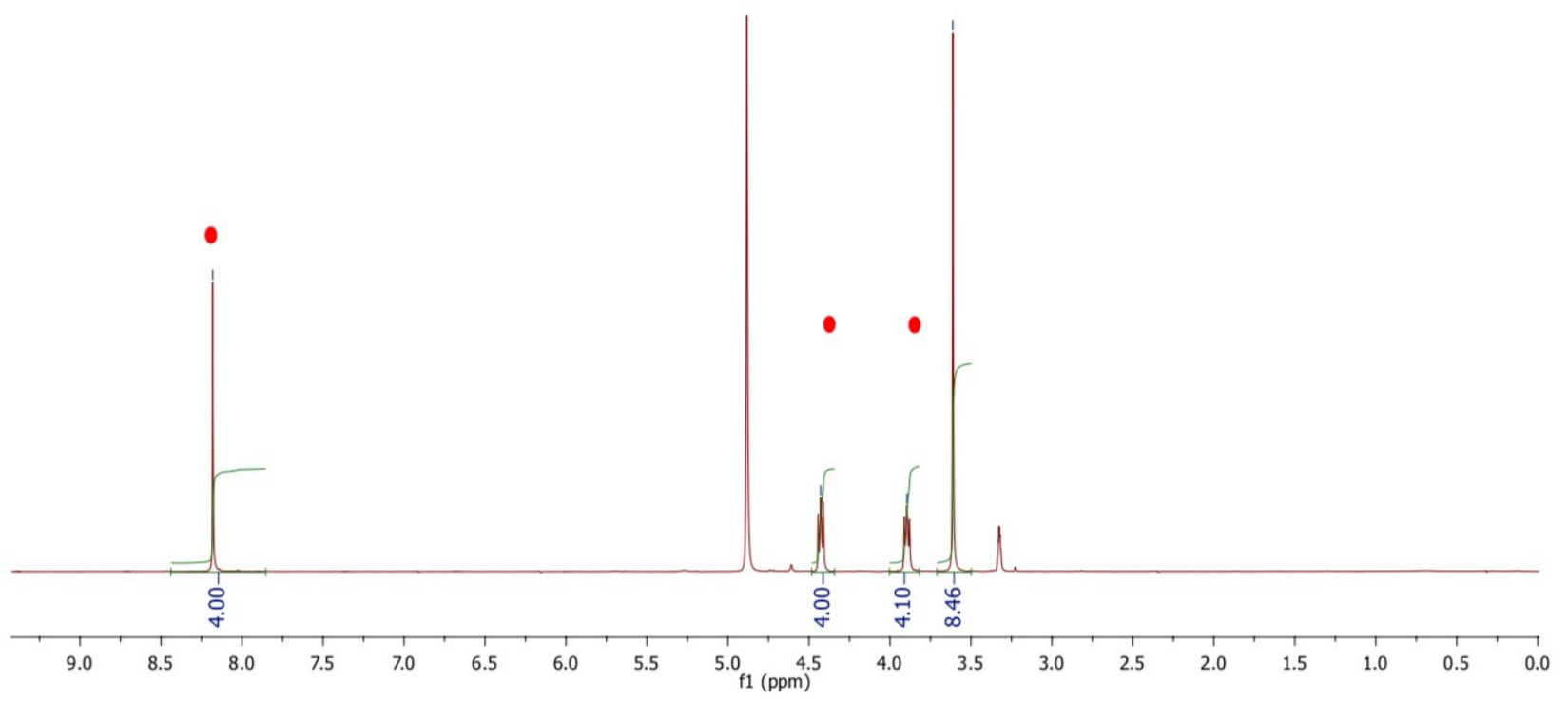

Figure S3 ${ }^{1} \mathrm{H}$ NMR spectra in $\mathrm{CD}_{3} \mathrm{OD} \_$of BHET obtained from glycolysis of post-consumer PET waste: a) colored transparent bottle, b) opaque bottle at $150{ }^{\circ} \mathrm{C}$, c) opaque bottle at $180{ }^{\circ} \mathrm{C}, \mathrm{d}$ ) bakery package at $150{ }^{\circ} \mathrm{C}$ and e) bakery package at $180^{\circ} \mathrm{C}$. Red dots mark the signals relevant to BHET. 
Table S1. Conversions, yields and reaction conditions for the glycolysis of PET at different temperatures.

\begin{tabular}{|c|c|c|c|c|c|}
\hline Catalyst & $\begin{array}{c}\text { Catalyst/polymer } \\
\text { mole ratio }\end{array}$ & $\mathbf{T}\left({ }^{\circ} \mathrm{C}\right)$ & t (h) & $\begin{array}{c}\text { Conversion } \\
(\%)\end{array}$ & $\begin{array}{l}\text { Yield } \\
(\%)\end{array}$ \\
\hline $\mathrm{TBA} \cdot 30 \mathrm{H}_{2} \mathrm{O}$ & $1: 5$ & 120 & 6 & 0 & 0 \\
\hline $\mathrm{TBA} \cdot 30 \mathrm{H}_{2} \mathrm{O}$ & $1: 5$ & 150 & 6 & 64 & 28 \\
\hline $\mathrm{TBA} \cdot 30 \mathrm{H}_{2} \mathrm{O}$ & $1: 5$ & 180 & 6 & 88 & 31 \\
\hline$[\mathrm{Ch}][\mathrm{Gly}]$ & $1: 5$ & 120 & 4 & 83 & 15 \\
\hline$[\mathrm{Ch}][\mathrm{Gly}]$ & $1: 5$ & 150 & 4 & 83 & 29 \\
\hline$[\mathrm{Ch}][$ Gly $]$ & $1: 5$ & 180 & 4 & 100 & 25 \\
\hline
\end{tabular}

Conversions and yields are reproducible within $\pm 5 \%$ based on triplicate runs.

Table S2. Conversions, yields and reaction conditions for the glycolysis of PET at different reaction times.

\begin{tabular}{|cccccc|}
\hline Catalyst & $\begin{array}{c}\text { Catalyst/polymer } \\
\text { mole ratio }\end{array}$ & $\mathbf{T ~}\left({ }^{\circ} \mathbf{C}\right)$ & $\mathbf{t ~ ( h )}$ & $\begin{array}{c}\text { Conversion } \\
\mathbf{( \% )}\end{array}$ & $\begin{array}{c}\text { Yield } \\
(\mathbf{\%})\end{array}$ \\
$\mathrm{TBA} \cdot 30 \mathrm{H}_{2} \mathrm{O}$ & $1: 5$ & 150 & 8 & 92 & 63 \\
& $1: 5$ & 150 & 6 & 80 & 43 \\
{$[\mathrm{Ch}][\mathrm{Gly}]$} & $1: 5$ & 150 & 8 & 90 & 38 \\
{$[\mathrm{Ch}][\mathrm{Gly}]$} & & & & & \\
\hline
\end{tabular}

Conversions and yields are reproducible within $\pm 5 \%$ based on triplicate runs. 
Table S3. Conversions, yields and reaction conditions for the glycolysis of PET at different catalyst loading.

\begin{tabular}{|c|c|c|c|c|c|}
\hline Catalyst & $\begin{array}{c}\text { Catalyst/polymer } \\
\text { mole ratio }\end{array}$ & $\mathbf{T}\left({ }^{\circ} \mathbf{C}\right)$ & t (h) & $\begin{array}{c}\text { Conversion } \\
(\%)\end{array}$ & $\begin{array}{l}\text { Yield } \\
(\%)\end{array}$ \\
\hline $\mathrm{TBA} \cdot 30 \mathrm{H}_{2} \mathrm{O}$ & $1: 10$ & 150 & 6 & 70 & 49 \\
\hline $\mathrm{TBA} \cdot 30 \mathrm{H}_{2} \mathrm{O}$ & $1: 7.5$ & 150 & 6 & 85 & 61 \\
\hline$[\mathrm{Ch}][\mathrm{Gly}]$ & $1: 10$ & 150 & 6 & 0 & 0 \\
\hline [Ch][Gly] & $1: 7.5$ & 150 & 6 & 49 & 23 \\
\hline
\end{tabular}

Conversions and yields are reproducible within $\pm 5 \%$ based on triplicate runs.

Table S4. Conversions, yields and reaction conditions for the glycolysis of PET promoted by different cholinium salts

\begin{tabular}{|c|c|c|c|c|c|}
\hline Catalyst & $\begin{array}{c}\text { Catalyst/polymer } \\
\text { mole ratio }\end{array}$ & $\mathbf{T}\left({ }^{\circ} \mathbf{C}\right)$ & t (h) & $\begin{array}{c}\text { Conversion } \\
(\%)\end{array}$ & $\begin{array}{l}\text { Yield } \\
(\%)\end{array}$ \\
\hline$[\mathrm{Ch}][\mathrm{Ala}]$ & $1: 5$ & 150 & 6 & 6 & 0 \\
\hline$[\mathrm{Ch}][\mathrm{Im}]$ & $1: 5$ & 150 & 6 & 9 & 0 \\
\hline$[\mathrm{Ch}][\mathrm{OH}]$ & $1: 5$ & 150 & 6 & 32 & 17 \\
\hline$[\mathrm{Ch}][\mathrm{Lys}]$ & $1: 5$ & 150 & 6 & 50 & 24 \\
\hline$[\mathrm{Ch}]_{3}\left[\mathrm{PO}_{4}\right]$ & $1: 5$ & 150 & 6 & 72 & 27 \\
\hline
\end{tabular}

Conversions and yields are reproducible within $\pm 5 \%$ based on triplicate runs. 